[Article]

\title{
黑色微弧氧化膜的制备及其表征
}

\author{
白晶莹 ${ }^{1, *}$ 李思振 ${ }^{1} \quad$ 郑大江 ${ }^{3} \quad$ 张立功 $^{1} \quad$ 冯 立 $^{1}$ \\ 崔庆新 ${ }^{1}$ 王景润 ${ }^{1} \quad$ 姜文武 ${ }^{1} \quad$ 林昌健 ${ }^{2, *}$ \\ ( ${ }^{1}$ 北京卫星制造厂, 中国空间技术研究院, 北京 100190; ${ }^{2}$ 厦门大学化学化工学院, 福建厦门 361005 ; \\ 3厦门大学材料学院, 福建厦门 361005)
}

\begin{abstract}
摘要: 利用二次微弧氧化法, 通过在磷酸盐体系的电解液中添加柠檬酸铁, 在 AZ40M 镁合金基底表面成功 制备了黑色的微弧氧化膜层。并利用扫描电子显微镜(SEM)、能谱仪(EDS)、X射线衍射(XRD)和 X射线光电 子能谱(XPS)分别对黑色膜层的形貌和成分组成进行了表征, 探讨了黑色微弧氧化膜层的形成过程及机理。 结果显示, 黑色膜层的主要成分为氧化镁, 同时伴随着一定量的铁氧化物, 包括四氧化三铁、氧化亚铁和单 质铁。因此镁基底表面黑色膜层的形成可归因于柠檬酸铁中的铁离子在成膜过程中参与反应形成了黑色的四 氧化三铁、氧化亚铁和单质铁混合物, 并均匀地沉积在氧化膜中。
\end{abstract}

关键词: 微弧氧化; 磷酸盐; 镁合金; 形成机理; 黑色氧化膜

中图分类号: O646; TG146

\section{Preparation and Characterization of Black Micro-Arc Oxidation Films}

\author{
BAl Jing-Ying ${ }^{1, *} \quad$ LI Si-Zhen ${ }^{1} \quad$ ZHENG Da-Jiang ${ }^{3} \quad$ ZHANG Li-Gong ${ }^{1} \quad$ FENG Li ${ }^{1}$ \\ CUI Qing-Xin ${ }^{1} \quad$ WANG Jing-Run ${ }^{1} \quad$ JIANG Wen-Wu ${ }^{1} \quad$ LIN Chang-Jian ${ }^{2, *}$ \\ ('Beijing Spacecrafts, China Academy of Space Technology, Beijing 100190, P. R. China; \\ ${ }^{2}$ College of Chemistry and Chemical Engineering, Xiamen University, Xiamen 361005, Fujian Province, P. R. China; \\ ${ }^{3}$ College of Materials, Xiamen University, Xiamen 361005, Fujian Province, P. R. China)
}

\begin{abstract}
Black micro-arc oxide film layers were prepared on AZ40M magnesium alloy substrates by twostep micro-arc oxidation in a phosphate electrolyte with ferric citrate. The morphologies and compositions of these films were characterized by scanning electron microscopy (SEM), energy-dispersive spectroscopy (EDS), X-ray diffraction (XRD), and X-ray photoelectron spectroscopy (XPS) to provide details regarding the film formation mechanism. The results showed that the main component of the oxide films was magnesium oxide, with various iron compounds also being present, including ferroferric oxide, ferrous oxide, and metallic iron. These results indicate that the formation of black film can be attributed to the precipitation of these species during the oxidation of iron ions by ferric citrate in the electrolyte.
\end{abstract}

Key Words: Micro-arc oxidation; Phosphate; Magnesium alloy; Formation mechanism; Black oxidation film

1 引 言

镁合金因其密度低，具有良好的导电导热特 性等, 已广泛应用于汽车、电子、通讯、国防及
航空航天等领域 1 。但由于金属镁的化学性质很活 泼, 在应用环境中的耐蚀性较差, 因此必须对其 表面进行防腐处理 ${ }^{2-5}$, 目前对镁合金表面处理的

Received: April 5, 2016; Revised: June 7, 2016; Published online: June 7, 2016.

*Corresponding authors. BAI Jing-Ying, Email: baijy1213@163.com. LIN Chang-Jian, Email: cjlin@xmu.edu.cn; Tel: +86-592-2189354.

The project was supported by the National Natural Science Foundation of China (21321062).

国家自然科学基金(21321062)资助项目

(c) Editorial office of Acta Physico-Chimica Sinica 
技术主要有化学转化 ${ }^{6,7}$ 、金属镀层 ${ }^{6,8}$ 、阳极氧 化 ${ }^{9-11}$ 、物理气相沉积 ${ }^{12,13}$ 和微弧氧化 ${ }^{14-18}$ 等。除了 提高耐蚀性, 在某些应用场合还需要考虑对镁合 金表面膜层进行着色，常见的着色方法有喷涂着 色、阳极氧化着色和金属镀层等, 但此类着色方 法得到的有色膜层往往与基底的结合力较弱, 在 使用过程中容易脱落。微弧氧化技术是继阳极氧 化技术发展起来的一种新型表面处理技术，通过 调节微弧氧化的电解液成分、氧化电压和氧化时 间等可得到不同性能的表面膜层, 而在电解液中 添加有色金属盐则可以使膜层显现出不同的颜 色, 且制备的膜层和基底的结合力较好 ${ }^{19-21}$ 。而目 前对镁合金微弧氧化的研究主要集中在优化各种 制备工艺，提高镁合金的耐蚀性方面 ${ }^{14,16,22,23}$, 而对 于氧化过程的着色机理和制备研究较少, 特别是 黑色氧化陶瓷膜的制备, 至今鲜见文献报道, 且 现有的氧化着色添加剂都以金属酸根为主 ${ }^{24,25}$, 使 得可制备的颜色较为受限。而黑色膜层是材料应 用中最为广泛的一种, 本工作主要利用铁离子作 为微弧氧化的着色添加剂, 在美合金表面制备完 全黑色的氧化膜, 并探讨微弧氧化制备黑色氧化 膜的电解液配方、氧化工艺及黑色膜层的形成机 理, 为镁合金微弧氧化表面着色技术及原理提供 参考。

\section{2 实验部分}

\section{1 镁合金微弧氧化膜的制备}

基体材料为 $\mathrm{AZ} 40 \mathrm{M}$ 镁合金(化学组成 $(w$, 质量 分数): $\mathrm{Al}, 3.0 \%-4.0 \% ; \mathrm{Zn}, 0.20 \%-0.80 \% ; \mathrm{Mn}$, $0.15 \%-0.50 \% ; \mathrm{Si}, \leq 0.10 \% ; \mathrm{Fe}, \leq 0.10 \% ; \mathrm{Cu}$, $\leq 0.05 \%$ ； $\mathrm{Be} ， \leq 0.01 \%$ ；其他元素， $\leq 0.30 \%$ ； $\mathrm{Mg}$ 为余量), 规格为 $10 \mathrm{~mm} \times 10 \mathrm{~mm} \times 1 \mathrm{~mm}$, 清 洗除油后, 放入微弧氧化的电解槽设备中, 接 好电极, 微弧氧化过程采用恒流模式, 电流密 度为 $2 \mathrm{~A} \cdot \mathrm{dm}^{-2}$, 占空比为 $20 \%-30 \%$, 在不同的 电解质溶液中氧化不同的时间, 获得不同的微弧 氧化膜层, 水洗、干燥, 备用。其中制备白色膜 层的微弧氧化溶液组成为: 磷酸钠 $(\geq 98.0 \%, 15$ $\left.\mathrm{g} \cdot \mathrm{L}^{-1}\right)$ 、氟化钠 $\left(\geq 98.0 \%, 3 \mathrm{~g} \cdot \mathrm{L}^{-1}\right)$ 及氢氧化钾 $\left(\geq 85.0 \%, 5.6 \mathrm{~g} \cdot \mathrm{L}^{-1}\right)$; 黑色膜层的微弧氧化溶液 组成为: 柠檬酸铁( $\mathrm{Fe}$ 含量: $16.5 \%-18.5 \%, 10 \mathrm{~g}$ · $\left.\mathrm{L}^{-1}\right)$ 、磷酸钠 $\left(\geq 98.0 \%, 20 \mathrm{~g} \cdot \mathrm{L}^{-1}\right)$ 及氟化钠 $(\geq$ $\left.98.0 \%, 5 \mathrm{~g} \cdot \mathrm{L}^{-1}\right)$ 。

\section{2 样品的性能及表征}

利用扫描电子显微镜(SEM, Hitachi S-4800, 日本日立公司)观察膜层的微观形貌, $X$ 射线能谱 仪(EDS, Hitachi S-4800, 日本日立公司)分析膜层 的元素组成, $X$ 射线衍射 $\left(X R D, X^{\prime}\right.$ pert PRO, 荷 兰帕纳科公司)分析膜层的相结构组成, $\mathrm{X}$ 射线光 电子能谱 (XPS, Quantum 2000, 美国 Physical Electronics 公司)分析元素的价态信息。

\section{3 结果与讨论}

\section{1 镁合金微弧氧化膜的制备}

在以磷酸盐为主盐的电解液溶液(磷酸钠、氟 化钠和氢氧化钾)中, 微弧氧化得到的氧化镁陶瓷 膜层为白色膜层, 见图 $1(\mathrm{~g})$ 内嵌数码图。通过 SEM 观察可知, 膜层为较均匀的多孔膜层, 孔径 大小约为 $1-2 \mu \mathrm{m}$, 孔隙周围为熔融状的小块固体 堆积而成, 侧面显示膜层相对较为致密, 并和基 体紧密结合在一起, 难以区分界面, 如图 1(a,b,c) 所示, 其中图 1(a) 是小倍数大范围观察, 图 1(b)为 局部区域的放大显微图, 图 1(c)为侧面形貌图。 EDS 测试结果显示膜层主要由 $\mathrm{C} 、 \mathrm{O} 、 \mathrm{Mg} 、 \mathrm{P}$ 元素 组成, 还有少量的 $\mathrm{F}$ 和 $\mathrm{Na}$ 元素, 说明电解质溶液 中的离子参与了微弧氧化膜层的生长过程, 尤其 是磷酸根和氟离子, 不仅参与了反应还成为了膜 层的一部分, 如图 $1(\mathrm{~g})$ 所示。而 XRD 测试结果显 示膜层主要由 $\mathrm{MgO}$ 组成, 还有少量的 $\mathrm{Mg}_{3}\left(\mathrm{PO}_{4}\right)_{2}$, 如图 2 所示, 镁合金样品中出现的峰都为金属 $\mathrm{Mg}$ 的特征峰, 强度最高的为 $\mathrm{Mg}$ 在 $32.2^{\circ} 、 34.4^{\circ}$ 和 $36.6^{\circ}$ 处的三重特征峰, 分别对应着金属 $\mathrm{Mg}$ 的 (100)、(002)和(101)晶面, 而表面制备了白色膜层 后, 除了 $\mathrm{Mg}$ 的特征峰, 在 $42.9^{\circ}$ 和 $62.4^{\circ}$ 处出现了 新的峰, 为 $\mathrm{MgO}$ 的特征峰, 对应着其(200)和(220) 晶面, 同时在 $33.0^{\circ}$ 处可看到一个很小的峰, 对应 于 $\mathrm{Mg}_{3}\left(\mathrm{PO}_{4}\right)_{2}$ 的 $(\overline{3} 11)$ 晶面特征峰, 与 $\mathrm{EDS}$ 结果相吻 合。

将制备有白色膜层的镁合金加入添加了柠檬 酸铁的电解液进行二次微弧氧化后, 形成的微弧 氧化膜层转变为黑色, 见图 1(h) 的内嵌数码图。 SEM 观察显示膜层表面有大小不等的孔洞, 直径 较大的可达到 $10 \mu \mathrm{m}$, 整体为致密的熔融物组成的 膜层, 且表面存在着明显的裂纹, 主要是由于高 压放电引起的熔融物在快速凝固过程中热应力过 大造成的, 而侧面 SEM 显示整个膜层内部呈现多 

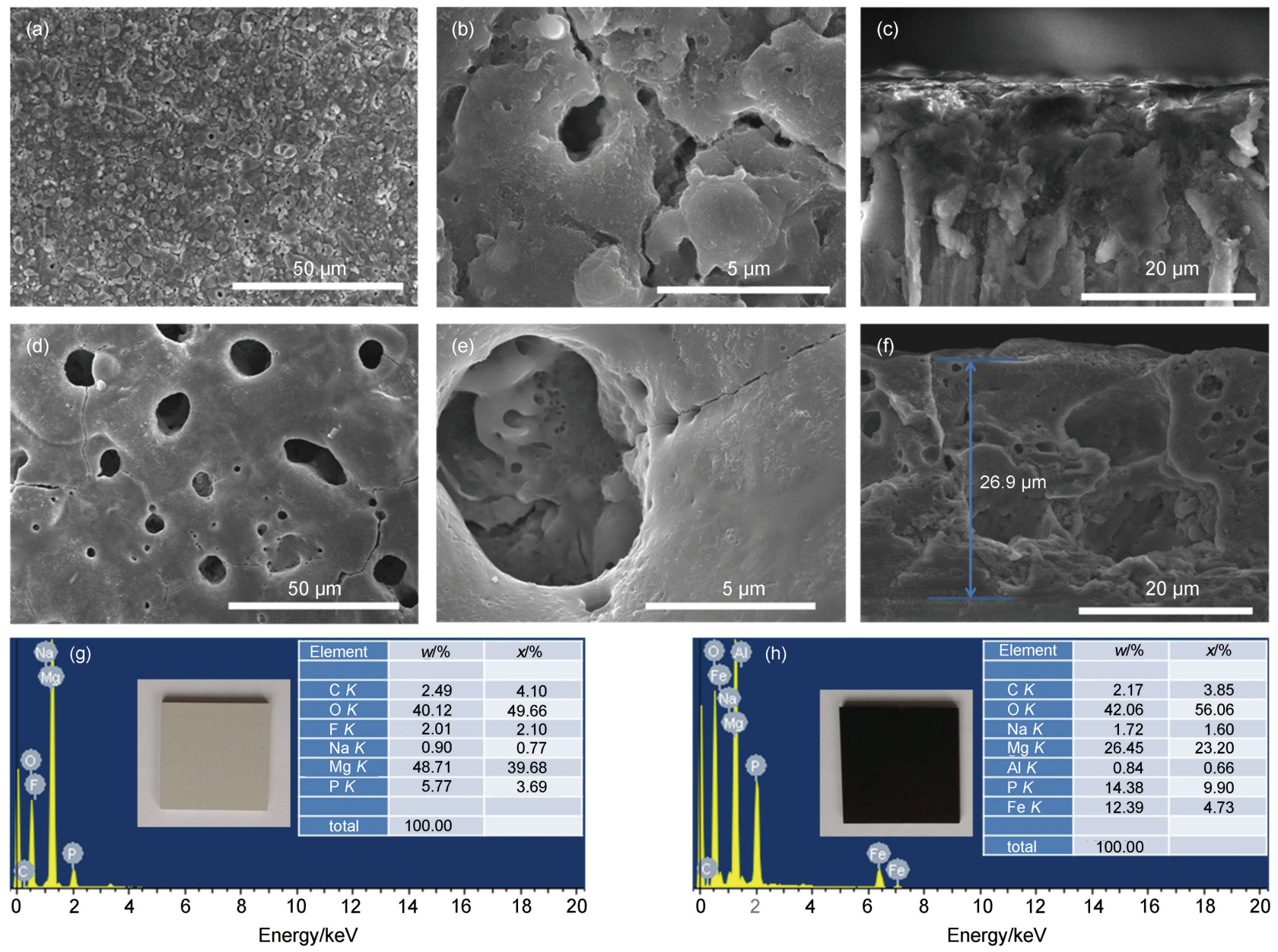

图 1 电解液中添加柠檬酸铁后二次微弧氧化对膜层表面形貌的影响

Fig.1 Effect on surface morphology of two-step micro-arc oxidation film after adding ferric citrate in electrolyte

SEM images of micro-arc oxidation film layer on surface of AZ40M magnesium alloy grown without additive ( $a, b, c)$ and with additive (d, e, f) after two-step micro-arc oxidation; EDS of micro-arc oxidation film layer grown without additive, the embedded is digital photograph of white film (g) and with additive after two-step micro-arc oxidation, the embedded is digital photograph of white film (h).

孔分布, 如图 1(d,e, f) 所示, 其中图 1(d) 是小倍数 大范围观察, 图 1(e)为局部区域的放大显微图, 图 1(f)为侧面图, 膜层与基底界面较为清晰, 膜层厚 度约为 $26.9 \mu \mathrm{m}$ 。 $\mathrm{EDS}$ 结果显示膜层主要由 $\mathrm{C} 、$ $\mathrm{O} 、 \mathrm{Mg} 、 \mathrm{P} 、 \mathrm{Fe}$ 元素组成, 还有少量的 $\mathrm{Al}$ 和 $\mathrm{Na}$ 元 素, 相比于白色膜层, 最大的差别在于多了 $\mathrm{Fe}$ 元 素的存在, $\mathrm{Al}$ 为镁合金自身所带, 而 $\mathrm{Na}$ 元素则来 自电解液溶液, 具体元素含量见图 1(h)所列。通过 EDS 测试分析膜层表面不同位置的元素含量发 现, 孔隙处 $\mathrm{Fe}$ 的原子百分含量相对于致密处要高 很多, 约增加了十几倍, 而在孔隙处 $\mathrm{Mg}$ 和 $\mathrm{O}$ 元素 的含量明显偏低, 见图3(a), 说明孔隙处的部分 $\mathrm{Fe}$ 元素极可能以单质的形式存在。同时在膜层侧面 不同致密区域进行 EDS 元素含量分析发现, Fe 元 素含量在膜层中梯度分布, 膜层表面含量最高,
越靠近镁合金基底， $\mathrm{Fe}$ 元素含量越低，其原子百 分比到达镁合金基底处时接近于 0 , 相关数据见图 3(b)。通过对其进行 XRD 测试发现, $\mathrm{Mg}$ 的基底峰 减弱, $\mathrm{MgO}$ 的特征峰增强, 主要是由于生成的微 弧氧化膜层相对较厚, 表层以氧化镁为主。相比 于白色膜层, 黑色膜层的 XRD 谱图中 $44.8^{\circ}$ 处出现 了一个微弱的小峰, 对应于四氧化三铁的(400)晶 面(PDF Reference code: 00-026-1136)。

为了验证 $\mathrm{Fe}$ 元素在膜层中的存在形式, 进行 了XPS 的测试, 图 4(a)为膜层表面的XPS 全谱扫描 图, 结果显示膜层表面的 $\mathrm{Mg}$ 和 $\mathrm{O}$ 元素的峰信号最 强, 说明膜层主要由 $\mathrm{MgO}$ 组成, $\mathrm{Fe}$ 元素信号较 弱, 说明相对含量比较低, 通过对 Fe $2 p$ 区域进行 窄谱扫描, 结果显示在表面致密处 $\mathrm{Fe}$ 元素主要以 三价和二价氧化物的形式存在, $\mathrm{Fe} 2 p_{1 / 2}$ 和 $\mathrm{Fe} 2 p_{3 / 2}$ 


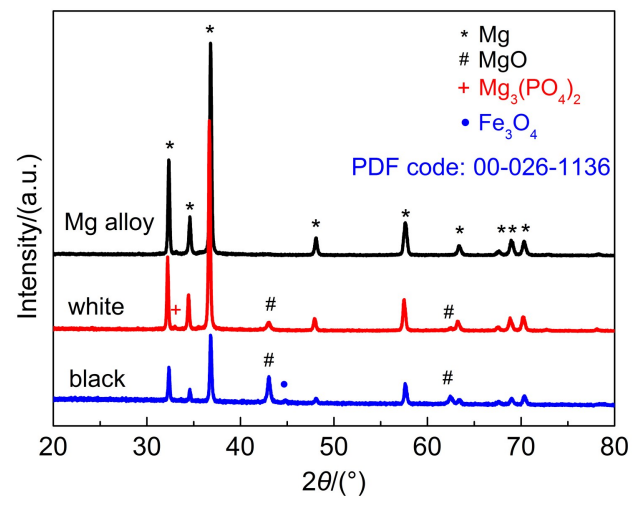

图 2 AZ40M镁合金和制备有不同表面膜层 镁合金的 XRD 图

Fig.2 XRD patterns of AZ40M magnesium alloys and different micro-arc oxidation film layer on surface of AZ40M magnesium alloy

的主峰位置分别出现在 723.58 和 $710.08 \mathrm{eV}$ 处, 与 $\mathrm{Fe}_{3} \mathrm{O}_{4}$ 的特征谱峰位置相对应, 且两个峰之间没有 出现卫星峰, 此为 $\mathrm{Fe}_{3} \mathrm{O}_{4}$ 区别于其他铁氧化物的一 个明显特征 ${ }^{26}$, 如图 4(b) 所示。通过对 $\mathrm{Fe} 2 p_{3 / 2}$ 峰进 行分峰拟合, 并计算峰面积发现, $\mathrm{Fe}^{2+}$ 与 $\mathrm{Fe}^{3+}$ 的比 例为: $1 / 1.97$, 接近于 $1 / 2$, 说明膜层表面的铁氧化 物主要是 $\mathrm{Fe}_{3} \mathrm{O}_{4}$, 如图 4(c) 所示。同时通过离子束 溅射到达 $500 \mathrm{~nm}$ 深度对膜层内部进行了 XPS 全谱
扫描, 相比于表面的全谱扫描图, C元素的特征峰 几乎消失, 说明表面的 $\mathrm{C}$ 元素主要是环境中的污染 所致, 而 $\mathrm{Fe}$ 元素的特征峰则明显增强, 如图 4(d) 所示。通过对 Fe $2 p$ 区域的窄谱扫描可知, Fe $2 p_{1 / 2}$ 和 $\mathrm{Fe} 2 p_{3 / 2}$ 的主峰位置略有往低能量区偏移, 且在 约 $715.50 \mathrm{eV}$ 处出现一个卫星峰, 对应于 $\mathrm{FeO}$ 的特 征峰 ${ }^{26}$, 在 $706.58 \mathrm{eV}$ 处出现一个明显的小峰, 对 应于单质 $\mathrm{Fe}$ 的 $2 p_{3 / 2}$ 特征峰 ${ }^{27}$, 见图 4(e)。对 $\mathrm{Fe} 2 p_{3 / 2}$ 峰进行分峰拟合, 并计算峰面积可知, $\mathrm{Fe}^{0} 、 \mathrm{Fe}^{2+}$ 和 $\mathrm{Fe}^{3+}$ 的比例约为 $0.05 / 1 / 0.9$, 说明膜层内除了 $\mathrm{Fe}_{3} \mathrm{O}_{4}$ 还存在 $\mathrm{FeO}$ 和单质铁, 见图 4(f)。

\section{2 氧化时间对形成微弧氧化膜的影响}

为了探究黑色膜层的形成过程, 考察了氧化 时间对微弧氧化膜层的影响，先在镁合金表面制 备一层如图 1 所示的白膜底层, 然后放入含有柠檬 酸铁添加剂的电解液中二次微弧氧化进行膜层颜 色的转化处理和生长。通过观察膜层的表观形貌 发现, 随着氧化时间的增加, 膜层由白变黄最后 变成黑色, 前 $20 \mathrm{~s}$ 基本没什么变化, 不过 $20 \mathrm{~s}$ 时开 始出现小黑斑点, $120 \mathrm{~s}$ 后膜层边缘优先黑化, 5 $\min$ 时已经看不到白斑, 但膜层泛黄, $10 \mathrm{~min}$ 后基 本变为黑色, 并逐渐加深。

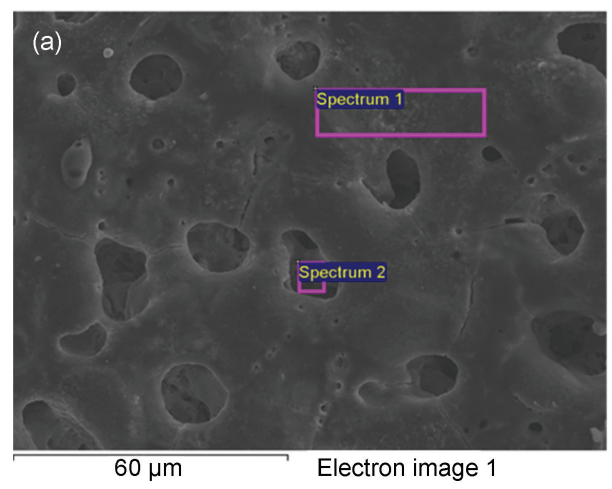

\begin{tabular}{|crr|}
\hline & \multicolumn{3}{|c|}{$x / \%$} \\
\hline Element & \multicolumn{1}{c}{1} & \multicolumn{1}{c|}{2} \\
\hline C K $K$ & 3.98 & \\
\hline Na K & 56.20 & 24.42 \\
\hline Mg K & 1.58 & 4.28 \\
\hline Al K & 22.93 & 10.63 \\
\hline P K & 0.65 & \\
\hline Fe K & 9.77 & 4.45 \\
\hline
\end{tabular}

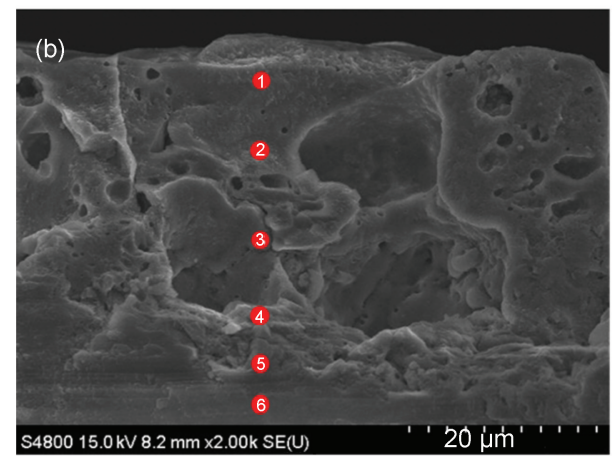

\begin{tabular}{|crrrrrr|}
\hline \multirow{2}{*}{ Element } & \multicolumn{7}{c|}{$x / \%$} \\
\cline { 2 - 7 } C K & \multicolumn{1}{c}{1} & \multicolumn{1}{c|}{2} & \multicolumn{1}{c|}{3} & \multicolumn{1}{c|}{5} & \multicolumn{1}{c|}{6} \\
\hline O K & 53.11 .18 & 55.54 & 51.41 & 51.88 & 45.04 & 26.73 \\
\hline Na K & 0.44 & 1.39 & 2.28 & 0.91 & 0.08 & 0.09 \\
\hline Mg K & 23.93 & 18.76 & 28.24 & 35.26 & 37.17 & 59.27 \\
\hline Al K & 0.75 & 0.35 & 0.85 & 0.89 & 1.04 & 2.05 \\
\hline P K & 8.70 & 9.99 & 6.30 & 3.68 & 0.88 & 0.65 \\
\hline Fe K & 3.68 & 2.80 & 1.20 & 1.18 & 0.68 & 0.11 \\
\hline
\end{tabular}

图3 黑色膜层在不同区域的 EDS 分析数据

Fig.3 EDS data analyses of micro-arc oxidation black film layer in different areas

(a) the surface and (b) cross-section of black film 

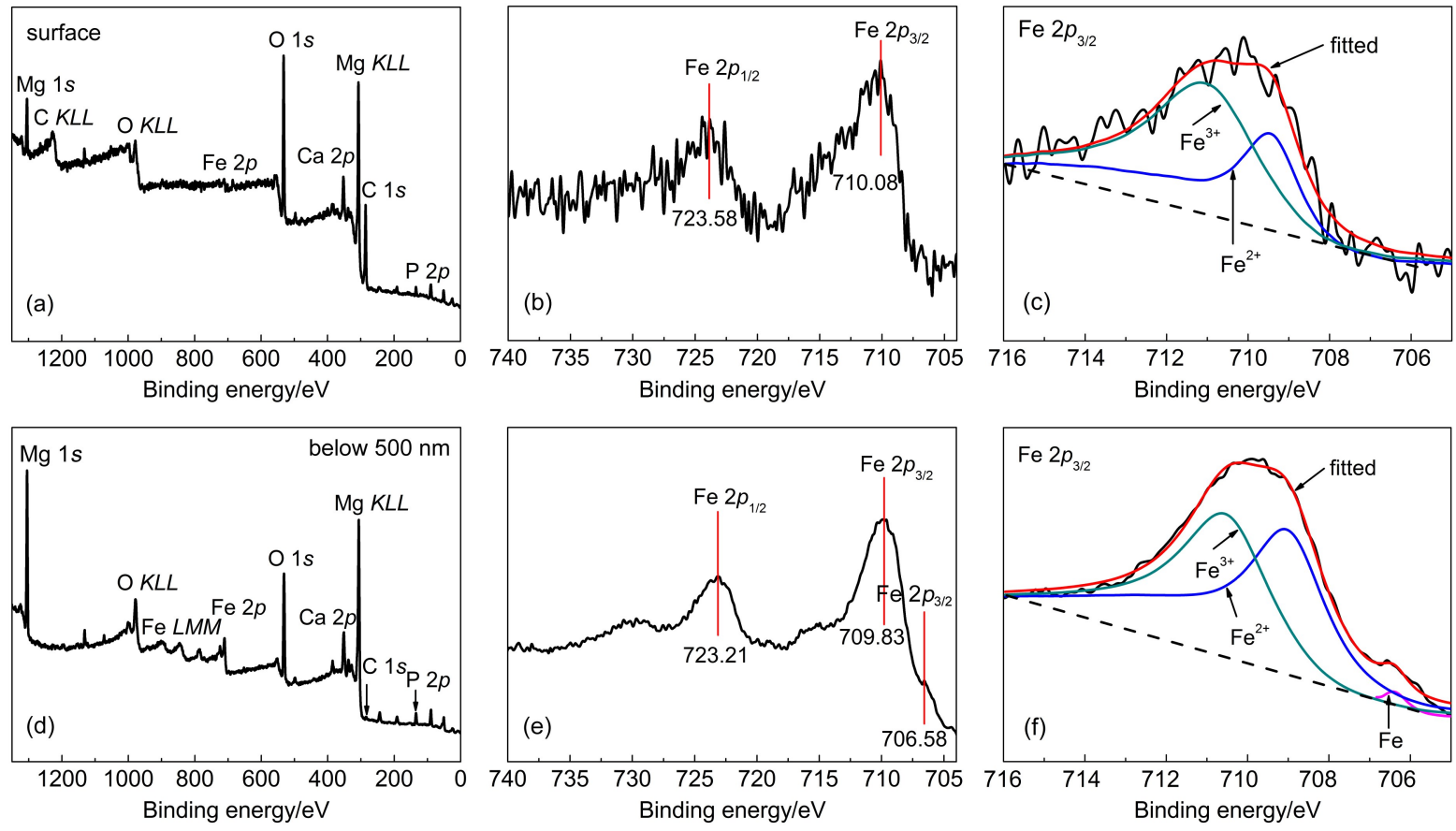

图4 微弧氧化黑色膜层表面的XPS 测试

Fig.4 XPS spectra of black film layer prepared by micro-arc oxidation on the surface of the film: (a) wide-scan spectrum, (b) Fe $2 p$, (c) fitted curve of Fe $2 p_{32}$; $500 \mathrm{~nm}$ below the surface: (d) wide-scan spectrum, (e) Fe $2 p$, (f) fitted curve of $\mathrm{Fe} 2 p_{3 / 2}$

通过 SEM 观察膜层表面形貌可发现, 刚开始 时样品表面呈碎片状的氧化膜, 与白色膜层的形 貌无明显差异, $10 \mathrm{~s}$ 后形貌开始发生变化, 出现了 大小不一的小孔, 孔径在几百纳米左右, 此时原 来的膜层已被击穿, $20 \mathrm{~s}$ 时微孔数量增加, 表面开 始出现熔融覆盖物, $40 \mathrm{~s}$ 时微孔继续增加, 表面出 现大量的致密的熔融物, 同时膜层出现裂纹, $70 \mathrm{~s}$ 后开始出现较大的微孔, 孔径大小约为 $1-2 \mu \mathrm{m}$, 且膜层表面沉积有大量的颗粒物, $120 \mathrm{~s}$ 后微孔继 续增加, 分布呈现均匀化, 大量的熔融物覆盖于 微孔上, $5 \mathrm{~min}$ 后出现更大的微孔, 周围沉积有大 量的熔融物, $10 \mathrm{~min}$ 后微孔膜开始均匀化, 微孔大 小从几微米到几十微米不等, $13 \mathrm{~min}$ 时膜层中微孔 明显, 周围为存在小孔和裂纹的熔融物, $16 \mathrm{~min}$ 时 微观形貌与 $13 \mathrm{~min}$ 时类似, 没有发生明显的变 化。图 5 给出了不同微弧氧化时间制备得到的膜层 的表面形貌 SEM 图, 其中图 5(A-I)为不同时间氧 化得到的样品的大范围低倍率形貌图, 图 5(a-i) 为 局部区域放大后的高倍率 SEM 形貌图, 从图中可 以显著地看到微弧氧化膜层的生长变化过程。

通过以上膜层微观形貌的变化可大致推断在 此条件下镁合金微弧氧化的主要过程: 短时间内 外加电压达到临界击穿电压, 白色膜层较为薄弱
的孔洞处优先被击穿, 形成导电小孔, 随着电压 继续升高, 镁合金基底处产生微弧放电, 此时放 电区域温度极高, 此处形成高温熔融物, 接触到 电解液时瞬间凝固沉积覆盖在膜层表面, 由于快 速凝固过程中热应力释放不足, 膜层表面形成裂 纹。此时膜层厚度增加, 阻抗增加, 外加电压继 续升高, 在膜层薄弱处再次击穿, 形成较大的微 孔, 伴随着的大量的热能使得美合金继续发生高 温氧化、熔融、凝固和沉积, 同时电解液中的离 子通过放电通道进入膜层内部和熔融物发生等离 子化学反应, 反应产物部分进入熔融物中, 部分 沉积在放电通道的内壁表面。随着氧化时间的增 长, 膜层不断在薄弱处放电击穿, 重新成膜, 逐 渐形成均匀的多孔膜层。

通过 EDS 元素分析发现, 微弧氧化初期 $\mathrm{Fe}$ 含 量随时间的增加而增加, $70 \mathrm{~s}$ 后基本保持不变, 只 是略有增加, 结合 SEM 微观形貌可知, 此时已形 成多孔膜层, 具体元素含量组成见表 1 。通过在膜 层表面不同区域进行 EDS 元素分析可知后期微孔 处的 $\mathrm{Fe}$ 元素含量远远高于致密熔融膜中, $70 \mathrm{~s}$ 前 不同区域差别不明显, 但膜层中可以明显发现底 膜中 $\mathrm{F}$ 元素的存在, 膜层破损处还有一定量的 $\mathrm{Mn}$ 元素, 为熔融基底喷射外溢的结果, $70 \mathrm{~s}$ 后白色底 

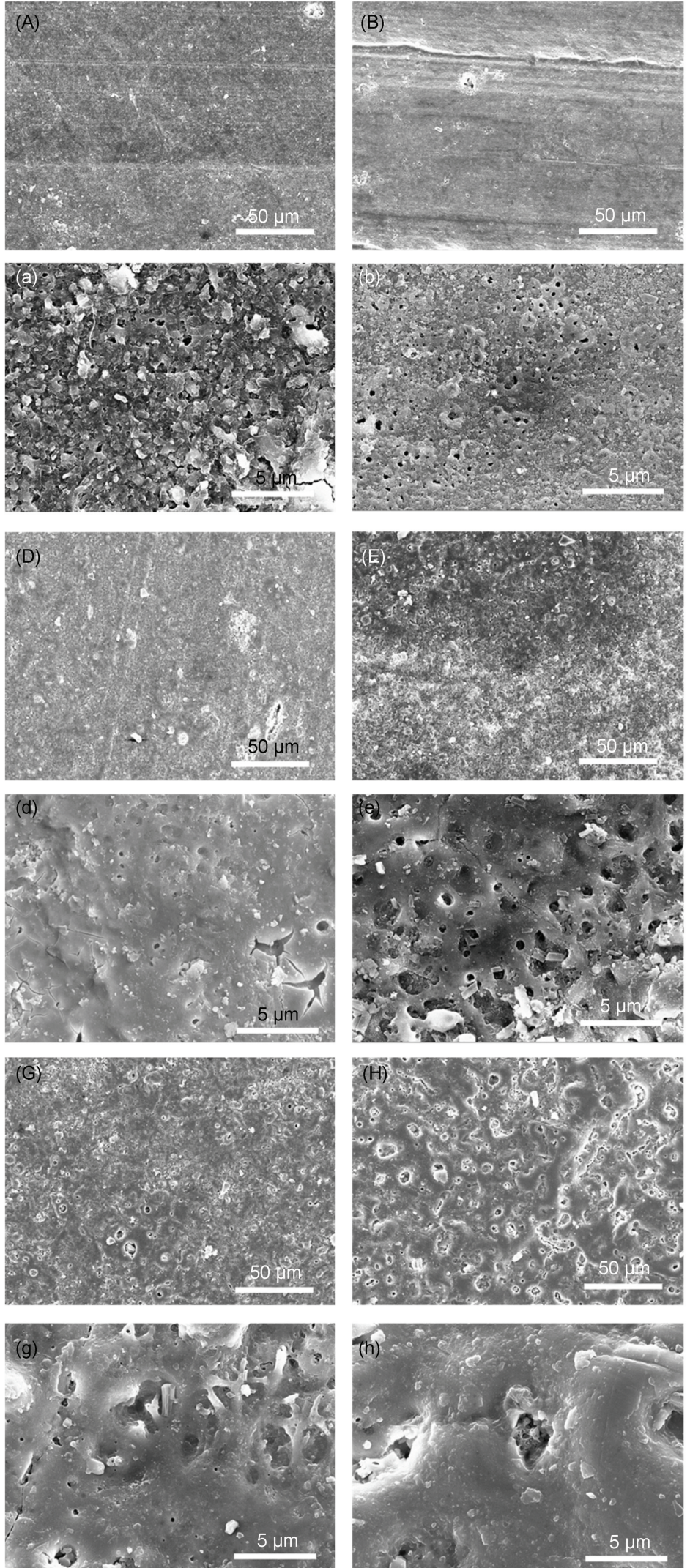
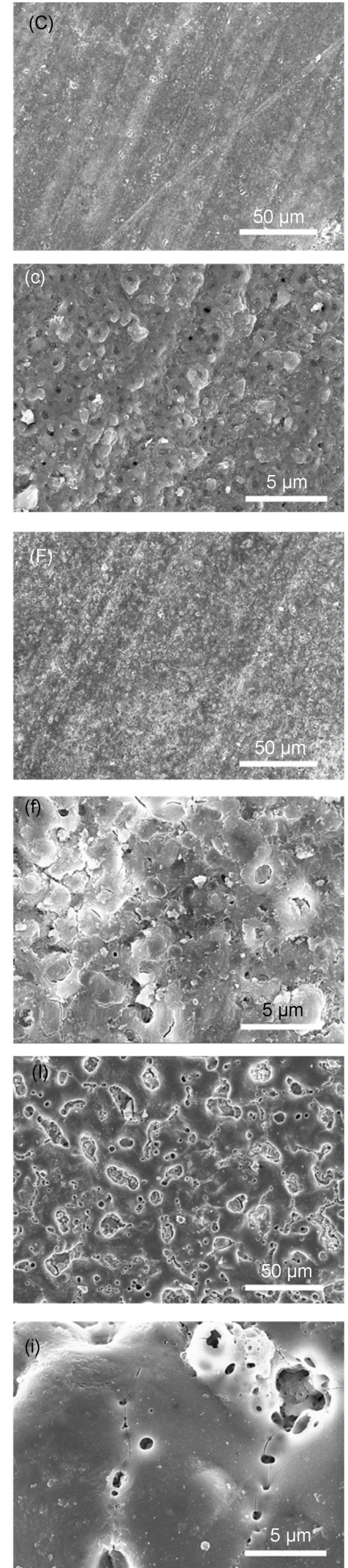

图 5 在含柠檬酸铁的电解液中二次微弧氧化不同时间制备得到的膜层表面 SEM 形貌

Fig.5 Surface SEM morphologies of films prepared by two-step micro-arc oxidation with different time in the electrolyte included ferric citrate

(A, a) $5 \mathrm{~s}$; (B, b) $10 \mathrm{~s}$; (C, c) $20 \mathrm{~s}$; (D, d) $40 \mathrm{~s}$; (E, e) $70 \mathrm{~s}$; (F, f) $120 \mathrm{~s}$; (G, g) $5 \mathrm{~min}$; (H, h) $10 \mathrm{~min}$; (I, i) $13 \mathrm{~min}$ 
表 1 在含柠檬酸铁的电解液中二次微弧氧化不同时间后得到的膜层主要组成元素含量变化

Table 1 Element contents of films prepared by two-step micro-arc oxidation with different time in the electrolyte included ferric citrate

\begin{tabular}{crrrrrrrrrr}
\hline \multirow{2}{*}{ Element } & \multicolumn{10}{c}{ Atomic fraction/\% } \\
\cline { 2 - 11 }$y$ & \multicolumn{1}{c}{$5 \mathrm{~s}$} & \multicolumn{1}{c}{$10 \mathrm{~s}$} & \multicolumn{1}{c}{$20 \mathrm{~s}$} & \multicolumn{1}{c}{$40 \mathrm{~s}$} & \multicolumn{1}{c}{$70 \mathrm{~s}$} & $120 \mathrm{~s}$ & $5 \mathrm{~min}$ & $10 \mathrm{~min}$ & $13 \mathrm{~min}$ & $16 \mathrm{~min}$ \\
\hline $\mathrm{C} K$ & 18.62 & 12.93 & 15.02 & 15.57 & 13.80 & 14.04 & 14.66 & 15.97 & 15.22 & 15.45 \\
$\mathrm{O} K$ & 38.07 & 41.09 & 43.02 & 54.03 & 54.55 & 53.15 & 52.53 & 54.39 & 48.77 & 53.24 \\
$\mathrm{Na} K$ & 0.63 & 0.40 & 0.46 & 1.24 & 2.10 & 1.95 & 2.59 & 3.97 & 2.71 & 2.52 \\
$\mathrm{Mg} K$ & 39.26 & 41.49 & 37.19 & 18.30 & 15.97 & 14.90 & 17.55 & 11.29 & 15.86 & 14.09 \\
$\mathrm{Al} K$ & 0.92 & 1.00 & 1.00 & 0.27 & 0.17 & 0.31 & 0.41 & 0.20 & 0.17 & 0.36 \\
$\mathrm{P} K$ & 2.45 & 2.93 & 3.18 & 8.33 & 9.92 & 10.72 & 9.03 & 10.30 & 13.12 & 10.04 \\
$\mathrm{Fe} K$ & 0.05 & 0.16 & 0.13 & 2.26 & 3.50 & 4.92 & 3.24 & 3.87 & 4.14 & 4.31 \\
\hline
\end{tabular}

膜被破坏重构, 基本不存在 $\mathrm{F}$ 元素, 取而代之的是 大量的 $\mathrm{Fe}$ 元素, 主要是电解液中的铁盐在高温反 应过程中部分通过等离子交换进入熔融膜层, 而 大部分成为铁氧化物或单质铁沉积在微孔通道内 壁，此类区域的 $\mathrm{Fe}$ 元素含量通常比致密区高十倍 以上。取 $5 \mathrm{~min}$ 之后的样品进行 XRD 表征, 图 6 结 果显示膜层主要由 $\mathrm{MgO}$ 组成, 其中还有少量的 $\mathrm{Fe}_{3} \mathrm{O}_{4}$, 而 $\mathrm{MgO}$ 的特征峰/ $\mathrm{Mg}$ 的特征峰的峰强度比 值随着氧化时间的增加明显增强，主要是归结于 膜层厚度的增加, $\mathrm{Mg}$ 基底峰的强度下降, $\mathrm{MgO}$ 的 特征峰强度增加, 而 $\mathrm{Fe}_{3} \mathrm{O}_{4}$ 的特征峰变化并不明 显, 说明 $\mathrm{Fe}_{3} \mathrm{O}_{4}$ 在膜层中的含量趋于稳定, 与 $\mathrm{EDS}$ 的数据是一致的。

\section{3 微弧氧化黑色膜层的形成机理探讨}

目前国内外对镁合金微弧氧化的成膜机理研 究认为, 一般分为三个阶段: 致密层生成阶段, 多孔层生成阶段和多孔层稳定生长阶段, 其中涉 及到两个重要的过程：一是高电压下致密氧化膜

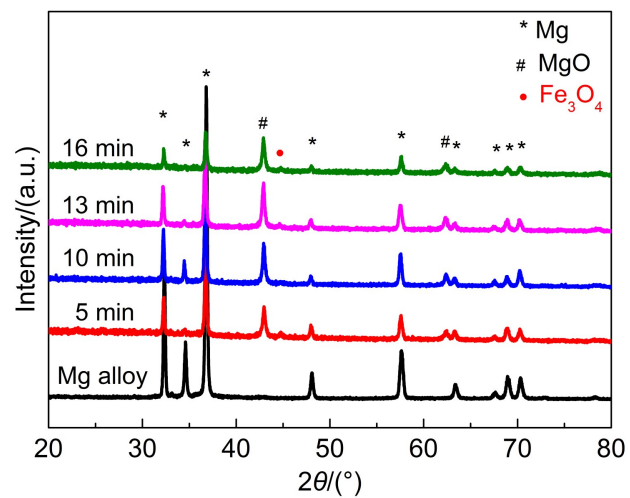

图 6 在含柠檬酸铁的电解液中二次微弧氧化不同时间 制备得到膜层的 XRD 图

Fig.6 XRD patterns of films prepared by two-step micro-arc oxidation with different time in the electrolyte included ferric citrate
击穿; 二是氧化膜微孔内气体在镁合金基底处的 微弧放电。在本实验条件下，采用两步氧化成膜 法, 首先在碱性磷酸盐体系中微弧氧化制备较为 致密的白色陶瓷底膜, 然后再将样品放入含有柠 檬酸铁添加剂的电解液中二次微弧氧化生长沉积 黑色膜层, 图 7 给出了镁合金基底表面膜层生长的 形成过程示意图。为保证第二次微弧氧化过程中 的顺利放电，第一次成膜制备的微弧氧化陶瓷膜 一般较薄，膜层表面微孔多而密集，作为接下来 二次微弧氧化微弧放电的导电通道和膜层内初始 电场的建立，为避免对后续着色的干扰，一般为 白色膜层，对应于图 7 中的第 I 阶段。因此将生长 有白色陶瓷底膜的样品放入含柠檬酸铁添加剂的 电解液中二次微弧氧化时，直接开始多孔层的生 长, 首先, 外加电压在 $20 \mathrm{~s}$ 内迅速上升到起弧电压 过程中将原始的白膜击穿，重新进入膜层生长阶 段，部分小孔成为新的导电通道，镁合金通过击 穿的小孔与溶液接触，高压下水被电解产生氧 气, 通过小孔析出, 当气体填满整个导电通道 后，电解液会被排除通道，从而与镁合金基底短 暂隔离，使得通道中的气体承受高压电场，产生

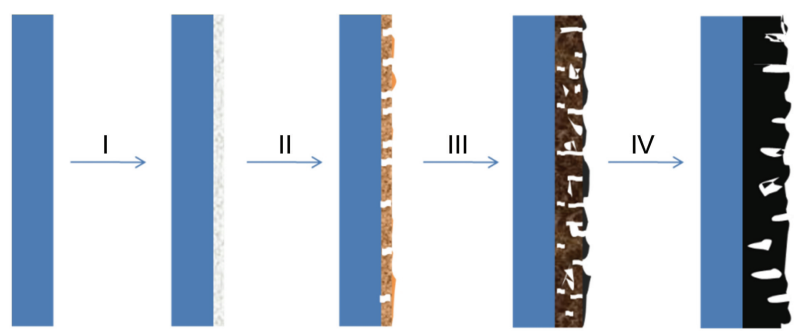

图 7 镁合金表面经二次微弧氧化生长黑色氧化膜层的 形成过程示意图

Fig.7 Schematic diagram of black oxide film layer formation process by two-step micro-arc oxidation on the surface of magnesium alloy 
电离, 当电压上升到起弧电压时产生微弧放电现 象。在放电通道区域的微弧放电会在瞬间释放大 量的热量, 导致其周边温度迅速上升, 发生镁合 金基底的剧烈氧化进一步增加热量的释放, 最终 造成氧化物膜层的熔融, 并喷射到膜层表面, 部 分在溶液相沉淀, 当气体被氧化反应消耗后, 微 弧放电暂时停止, 熔融态氧化物在电解液界面处 迅速冷却, 覆盖于膜层表面, 完成一次微弧氧化 过程中的膜层生长, 如图 5(a-d) 所示, 属于微弧 氧化的重新开始阶段, 膜层开始发黄, 对应于图 7 中的第 II 阶段。

随着膜层厚度增加, 前期的小孔放电通道逐 渐封闭, 膜层薄弱处优先被击穿形成新的导电和 放电通道, 但数量减少, 尺寸增加, 此时膜层的 均匀性下降, 微孔分布极不均匀, 且大小不一, 不过膜层总是在相对薄弱的区域被优先击穿、放 电、成膜, 持续一段时间后, 使得整个膜层逐渐 均匀化并且增厚, 微弧氧化的稳定电压也缓慢升 高, 如图 5(e-g) 所示, 属于多孔膜的生成阶段, 此时膜层中 $\mathrm{Fe}$ 沉积量随膜层的增厚而增加, 膜层 慢慢从黄色转变为黑色, 对应于图 7 中的第 III 阶 段。当进一步增加微弧氧化时间时, 氧化电压开 始明显增加, 用于击穿更厚的膜层以及维持更剧 烈的微弧放电, 此时微弧放电形成的微孔变深, 尺寸进一步增大, 密度降低, 而放电反应过程中 的热量释放更加强烈, 膜层的融合区域也变得更 大, 在迅速冷却凝固过程中, 由于热应力无法及 时释放, 使得熔融物沉积表面出现微裂纹, 膜层 厚度进一步增加, 如图 5(h, i) 所示, 处于多孔膜的 稳定生长阶段, 膜层基本呈黑色, 对应于图 7 中的 第 IV 阶段。

一般氧化膜的颜色取决于膜层成分中的金属 氧化物或其盐类, 本实验条件下形成的氧化膜成 分主要为氧化镁和铁氧化物, 其中氧化镁为白色 固体, 铁氧化物中氧化亚铁与四氧化三铁都呈黑 色, 结合上节 XRD 和 XPS 分析, 其铁氧化物可确 定为四氧化三铁和氧化亚铁的混合物。结合微弧 氧化的成膜过程和膜层的微观形貌及元素分析, 推测四氧化三铁和氧化亚铁在膜层中的掺杂过程 如下: 电弧放电过程中, 电解液中的三价铁离子 $\left(\mathrm{Fe}^{3+}\right)$ 会以铁盐络合物的形式通过放电通道进入膜 层内部, 在高温下与膜层熔融物发生化学反应, 形成化合物渗透入膜层内部, 影响膜层的成分组
成和微观结构, 从而影响膜层的颜色变化以及其 他性能等, 而金属离子还会被高温氧化或还原后 直接沉积在放电通道内部, 造成金属氧化物或金 属在微孔内的聚集。本实验中柠檬酸铁作为电解 液的添加剂, 其中的 $\mathrm{Fe}^{3+}$ 离子以电中性的络合物形 式存在于溶液中, 均匀分散在电解液中, 不受电 场影响发生迁移, 当沉积有白色底膜的镁合金样 品放入电解液中时, $\mathrm{Fe}^{3+}$ 溶液一起渗透进入或吸附 于膜层表面, 在微弧氧化过程中的高温环境中, 镁合金样品表面处会发生如下反应:

$$
\begin{aligned}
& \mathrm{Mg}+\mathrm{H}_{2} \mathrm{O} \rightarrow \mathrm{MgO}+\mathrm{H}_{2} \uparrow \\
& 2 \mathrm{Mg}+\mathrm{O}_{2} \rightarrow 2 \mathrm{MgO} \\
& 2 \mathrm{Fe}\left(\mathrm{C}_{6} \mathrm{H}_{5} \mathrm{O}_{7}\right)+9 \mathrm{O}_{2} \rightarrow \mathrm{Fe}_{2} \mathrm{O}_{3}+12 \mathrm{CO}_{2}+5 \mathrm{H}_{2} \mathrm{O} \\
& \mathrm{Fe}_{2} \mathrm{O}_{3}+\mathrm{Mg} / \mathrm{Al} \rightarrow \mathrm{Fe}+\mathrm{Mg} / \mathrm{AlO}_{x} \\
& \mathrm{Fe}_{2} \mathrm{O}_{3}+3 \mathrm{H}_{2} \rightarrow 2 \mathrm{Fe}+3 \mathrm{H}_{2} \mathrm{O} \\
& 3 \mathrm{Fe}+2 \mathrm{O}_{2} \rightarrow \mathrm{Fe}_{3} \mathrm{O}_{4} \\
& 3 \mathrm{Fe}+4 \mathrm{H}_{2} \mathrm{O}(\mathrm{g}) \rightarrow \mathrm{Fe}_{3} \mathrm{O}_{4}+4 \mathrm{H}_{2} \uparrow
\end{aligned}
$$

首先镁合金基底的金属 $\mathrm{Mg}$ 会和水汽或氧气在 高温下发生(1)和(2)反应, 生成 $\mathrm{MgO}$, 成为微弧氧 化膜层的主要组成部分, 同时溶液中的柠檬酸铁 在高温下发生分解(3)形成氧化铁, 而形成的氧化 铁在高温下接触镁合金会发生强烈的镁热或铝热 反应(4)从而被还原成单质铁。另一方面 $\mathrm{Mg}$ 和水反 应产生的氢气在高温下与氧化铁接触也会使其转 化为单质铁(5), 而反应(4)和(5)生成的单质铁在高 温下接触氧气和水蒸汽时会转化为四氧化三铁(6) 和(7)。因此部分 $\mathrm{Fe}^{3+}$ 通过微孔通道进入膜层内部, 在高温下形成黑色的 $\mathrm{Fe}_{3} \mathrm{O}_{4}$ 进入熔融物, 部分在高 温水汽环境中被 $\mathrm{Mg}$ 或 $\mathrm{H}_{2}$ 还原成单质 $\mathrm{Fe}$ 沉积在膜 层微孔中, 同时以上还原单质铁的过程中如还原 不完全则会形成部分氧化亚铁存在于膜层中。此 过程中 $\mathrm{Fe}^{3+}$ 离子浓度对多孔膜的生长速度以及微孔 结构影响甚大, 浓度的增加可明显增加镁合金的 成膜速率, 在氧化初期, 膜层中形成的铁氧化物 使得膜层发黑, 膜层中铁氧化物含量越高, 发黑 程度也越高, 当膜层达到一定厚度后, 铁氧化物 的浓度则不再是影响膜层发黑程度的主要因素。 随着微弧氧化时间的增加, 膜层增厚的同时膜层 中的铁氧化物含量也随之增加, 因此发黑程度也 随之增加, 达到一定程度后形成完全黑色的膜层。

\section{4 结 论}

本文利用三价铁盐作为镁合金微弧氧化电解 
液的添加剂, 制备了具有均匀黑色特征的镁合金 微弧氧化膜, 这主要是由于 $\mathrm{Fe}^{3+}$ 在成膜过程中形成 黑色的四氧化三铁、氧化亚铁和单质铁沉积在膜 层内部, 使得膜层发黑, 其中 $\mathrm{Fe}^{3+}$ 以柠檬酸络合盐 的形式存在溶液中至关重要, 使得 $\mathrm{Fe}^{3+}$ 可均匀吸附 在膜层表面并通过微孔进入到膜层内部, 随着氧 化时间的增加, 膜层会逐渐增厚, 膜层中的微孔 尺寸也会逐渐变大, 孔径加深, 从而增加膜层的 发黑程度。

\section{References}

(1) Kulekci M. K. Int. J. Adv. Manuf. Tech. 2008, 39 (9-10), 851. 10.1007/s00170-007-1279-2

(2) Song, G. L.; Atrens, A. Adv. Eng. Mater. 1999, 1 (1), 11. doi: 10.1002/(SICI)1527-2648(199909)1:1<11::AID-ADEM11> 3.0.CO;2-N

(3) Gray, J. E.; Luan, B. J. Alloy. Compd. 2002, $336(1-2), 88$. doi: 10.1016/S0925-8388(01)01899-0

(4) Song, G. L. Adv. Eng. Mater. 2005, 7 (7), 563. doi: 10.1002/ adem.200500013

(5) Huang, R.; Chen, M. A.; Lu, X. B. Acta Phys. -Chim. Sin. 2011, 27 (1), 113. [黄荣, 陈明安, 路学斌. 物理化学学报, 2011, 27 (1), 113.] doi: 10.3866/PKU.WHXB20110113

(6) Huo, H. W.; Li, Y.; Wang, F. H. Corros. Sci. 2004, 46 (6), 1467. doi: 10.1016/j.corsci.2003.09.023

(7) Dabala, M.; Brunelli, K.; Napolitani, E.; Magrini, M. Surf. Coat. Tech. 2003, 172 (2-3), 227. doi: 10.1016/S0257-8972(03) 00336-0

(8) Ambat, R.; Zhou, W. Surf. Coat. Tech. 2004, 179 (2-3), 124. doi: 10.1016/S0257-8972(03)00866-1

(9) Song, G. L.; Shi, Z. Corros. Sci. 2014, 85, 126. doi: 10.1016/j. corsci.2014.04.008

(10) Veys-Renaux, D.; Barchiche, C. E.; Rocca, E. Surf. Coat. Tech. 2014, 251, 232. doi: 10.1016/j.surfcoat.2014.04.031

(11) Liu, Y.; Wei, Z. L.; Yang, F. W.; Zhang, Z. Acta Phys. -Chim. Sin. 2011, 27 (10), 2385. [刘妍, 卫中领, 杨富巍, 张 昭. 物 理化学学报, 2011, 27 (10), 2385.] doi: 10.3866/PKU. WHXB20110931
(12) Hollstein, F.; Wiedemann, R.; Scholz, J. Surf. Coat. Tech. 2003, 162 (2-3), 261. doi: 10.1016/S0257-8972(02)00671-0

(13) Wu, G. S. Mater. Lett. 2007, 61 (18), 3815. doi: 10.1016/j. matlet.2006.12.082

(14) Duan, H. P.; Du, K. Q.; Yan, C. W.; Wang, F. H. Electrochim. Acta 2006, 51 (14), 2898. doi: 10.1016/j.electacta.2005.08.026

(15) Guo, H. F.; An, M. Z. Appl. Surf. Sci. 2005, 246 (1-3), 229. doi: 10.1016/j.apsusc.2004.11.031

(16) Cui, X.; Lin, X.; Liu, C. Corros. Sci. 2015, 90, 402. doi: 10.1016/j.corsci.2014.10.041

(17) Chang, L. R.; Cao, F. H.; Cai, J. S.; Liu, W. J.; Zheng, J. J.; Zhang, J. Q.; Cao, C. N. Acta Phys. -Chim. Sin. 2012, 28 (1), 127. [常林荣, 曹发和, 蔡景顺, 刘文娟, 郑俊军, 张鉴清, 曹楚 南. 物理化学学报, 2012, 28 (1), 127.] doi: 10.3866/PKU. WHXB201111112

(18) Gou, Y. N.; Zhi, Y.; Zhang, D. F.; Shui, L. K.; Zhang, C. Y. J. Funct. Mater. 2015, 23 (46), 23012. [沟引宁, 直 研, 张丁非, 水路凯, 张春艳. 功能材料, 2015, 23 (46), 23012.]

(19) Ling, C.; Jing, H.; Yu, S. X. Rare Metals 2006, 25 (6, Suppl. 2), 146. doi: 10.1016/S1001-0521(08)60070-4

(20) Liu, J. A.; Zheng, Z. B.; Li, J. Q.; Zhu, X. Y.; Liu, Y.; Bi, G. L. Mater. Res. Innov. 2015, 19 (Suppl. 1), S79. doi: 10.1179/ 1432891715Z.0000000001373

(21) Shao, Z. C.; Zhang, Q. F.; Yang, L.; Wang, M.; Gao, H. Mater Manuf. Process. 2015, 30 (12), 1505. doi: 10.1080/ 10426914.2015.1004688

(22) Liu, L. L.; Yang, P. X.; Su, C. N.; Guo, H. F.; An, M, Z. Int. J. Electrochem. Sci. 2013, 8 (5), 6077.

(23) Wang, M. J.; Li, C. F.; Yen, S. K. Corros. Sci. 2013, 76, 142. doi: 10.1016/j.corsci.2013.06.037

(24) Chen, X. M.; Luo, C. P.; Liu, J. W. Mater. Rev. 2009, 23, 535. [陈显明, 罗承萍, 刘江文. 材料导报. 2009, 23, 535.]

(25) Wang, S.; Yan, F. Y. Nonferrous Metals Engineering 2014, 3, 36. [王 胜, 阎峰云. 有色金属工程, 2014, 3, 36.]

(26) Yamashita, T.; Hayes, P. Appl. Surf. Sci. 2008, 254 (8), 2441. doi: 10.1016/j.apsusc.2007.09.063

(27) Biesinger, M. C.; Payne, B. P.; Grosvenor, A. P.; Lau, L. W.; Gerson, A. R.; Smart, R. Appl. Surf. Sci. 2011, 257, 2717. doi: 10.1016/j.apsusc.2010.10.051 